\title{
Children, enfants à Rodman Hall
}

"Chaussettes vertes et bonne nuit, les étoiles / Green Socks and Goodnight Stars" was primarily an exhibition for francophone and French-learning children and it was therefore very important for us to be able to welcome young visitors.

The Niagara Region is home to a large francophone population, which nevertheless remains a minority group. For instance, and although the region counts several francophone schools, Brock University is resolutely anglophone; many francophones leave the area to study at bilingual universities in Ontario. The local job market offers few opportunities for speakers of French. Most cultural events are of course in English.

We extended invitations to several francophone and immersion schools in the region to visit the exhibition and participate in creative workshops over reading week at Brock. About 177 children from five different schools came to Rodman Hall. These schools are Ecole Ste Marguerite Bourgeoys and Dalewood French Immersion Public School, both in St. Catharines, Ecole Nouvel Horizon and Ecole Saint François d'Assise, both in Welland, and St. Mark, Beamsville.

Each group (6 in total) made an illustrated booklet using the stories written by students in the course "Children's Literature in French." Each child was given the responsibility of illustrating one page. Our instructions were: cover the entire page with drawings / use colors (reading week is in the middle of February when everything is grey, white and frozen outside) / draw at least one element from the text written at the bottom of the page / draw as many things you wish that are not in the text.

Below are included a small sample of children's illustrations as well as the texts in French written by the students of Children's literature.

« Chaussettes vertes et bonne nuit, les étoiles / Green Socks and Goodnight Stars » était en priorité une exposition pour enfants francophones ou qui apprennent le français. Il nous était donc très important de pouvoir accueillir un jeune public.

La région du Niagara abrite une large population francophone qui reste, néanmoins, une minorité. Par exemple, et bien que la région compte de nombreuses écoles francophones, l'Université Brock est résolument anglophone ; de nombreux francophones quittent la région pour étudier dans des universités bilingues de l'Ontario. Localement, le marché du travail offre peu de perspectives aux locuteurs francophones. La plupart des événements culturels sont bien sûr en anglais.

Nous avons envoyé une invitation à plusieurs écoles francophones et d'immersion pour venir visiter l'exposition et participer à des ateliers de création durant la semaine de lecture à Brock. Environ 177 enfants de cinq écoles différentes sont venus à Rodman Hall. Ces écoles sont l'école Ste Marguerite Bourgeoys et Dalewood French Immersion Public School, toutes deux à St. Catharines, l'école Nouvel Horizon et l'école Saint François d'Assise, toutes deux à Welland, et St. Mark à Beamsville.

Chaque groupe (6 au total) a composé un livret illustrant les histoires rédigées par les étudiants du cours «Children's Literature in French ». Chaque enfant était responsable de l'illustration d'une page. Les instructions étaient : couvrir toute la page de dessins / utiliser des couleurs (la semaine de lecture à lieu mi-février lorsque tout est gris, blanc et gelé à l'extérieur) / dessiner au moins un élément du texte écrit au bas de la page / dessiner autant de choses qu'on veut qui ne sont pas dans le texte. 
Ci-dessous sont présentés un petit échantillon d'illustrations faites par les enfants, ainsi que les textes en français créés par les étudiants de Littérature pour enfants.

$\mathbf{C P}$

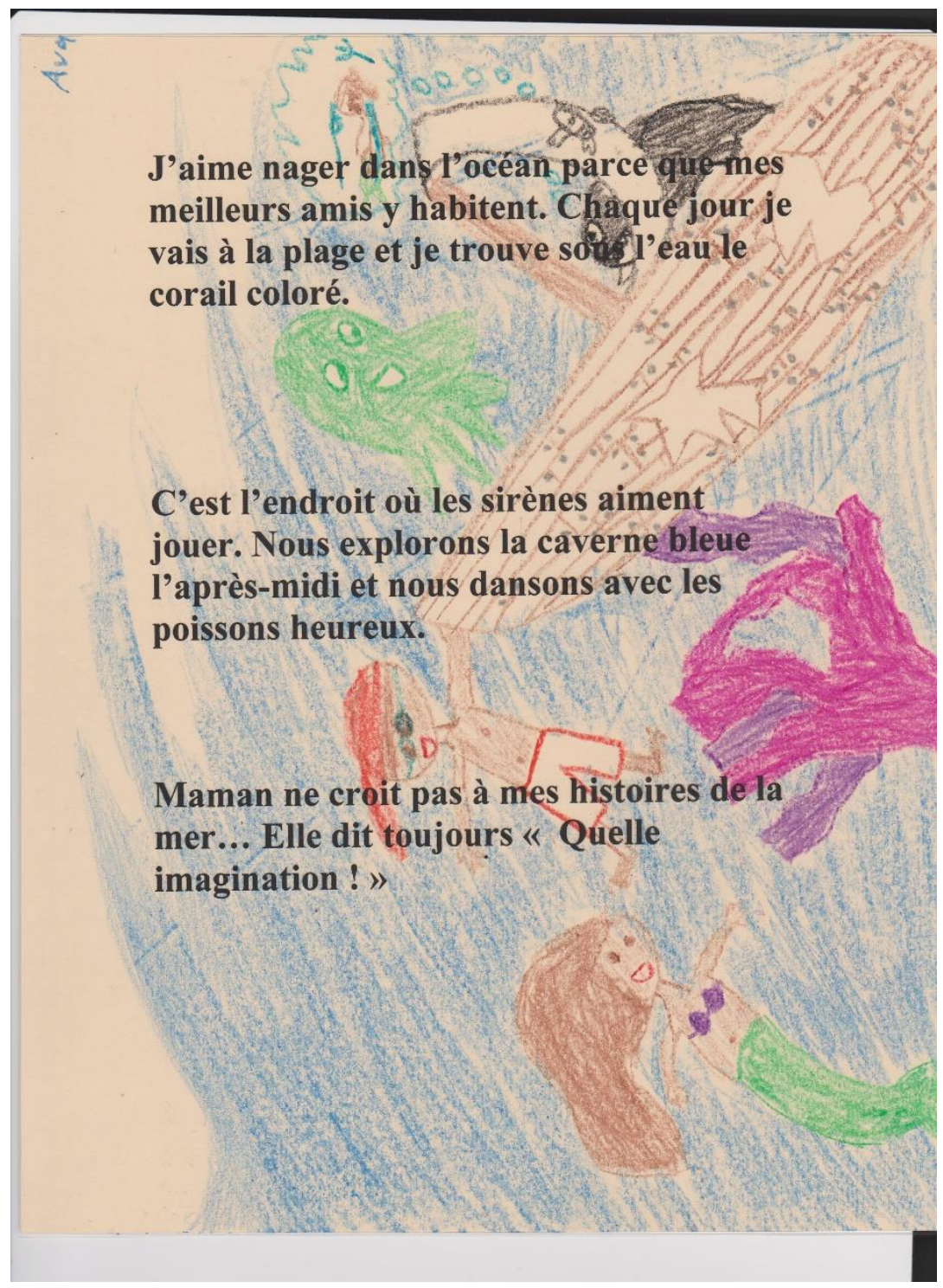



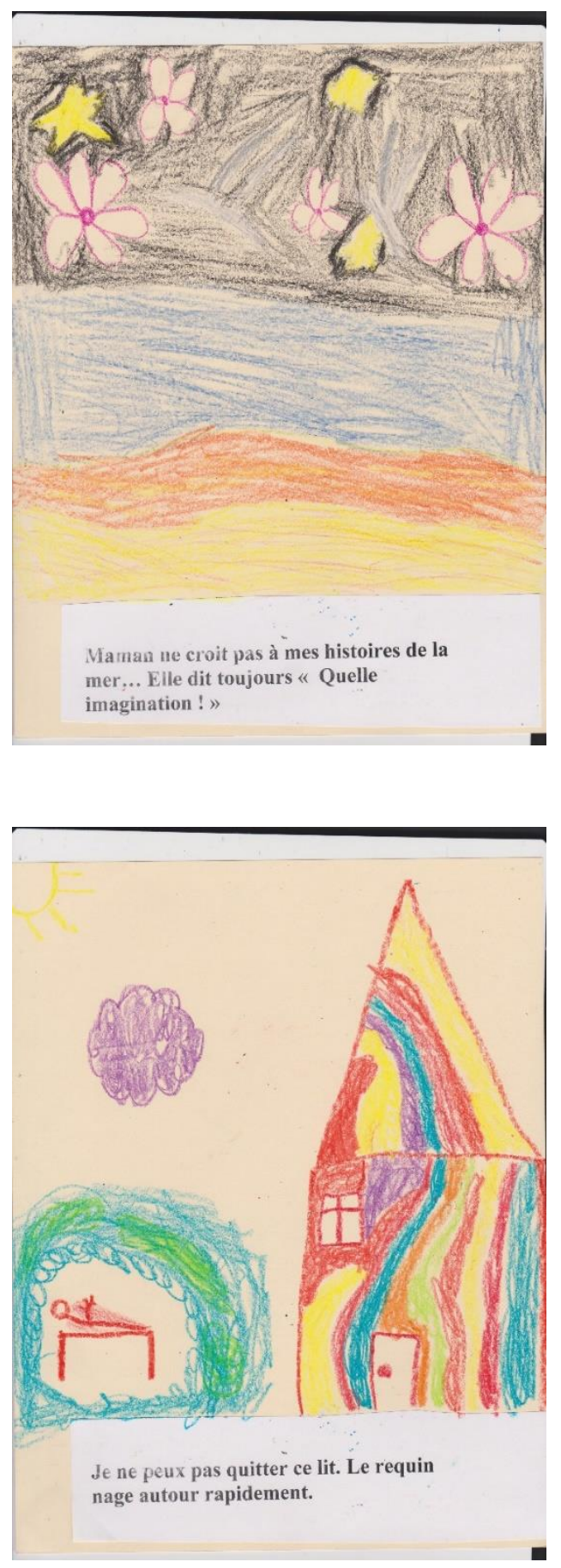
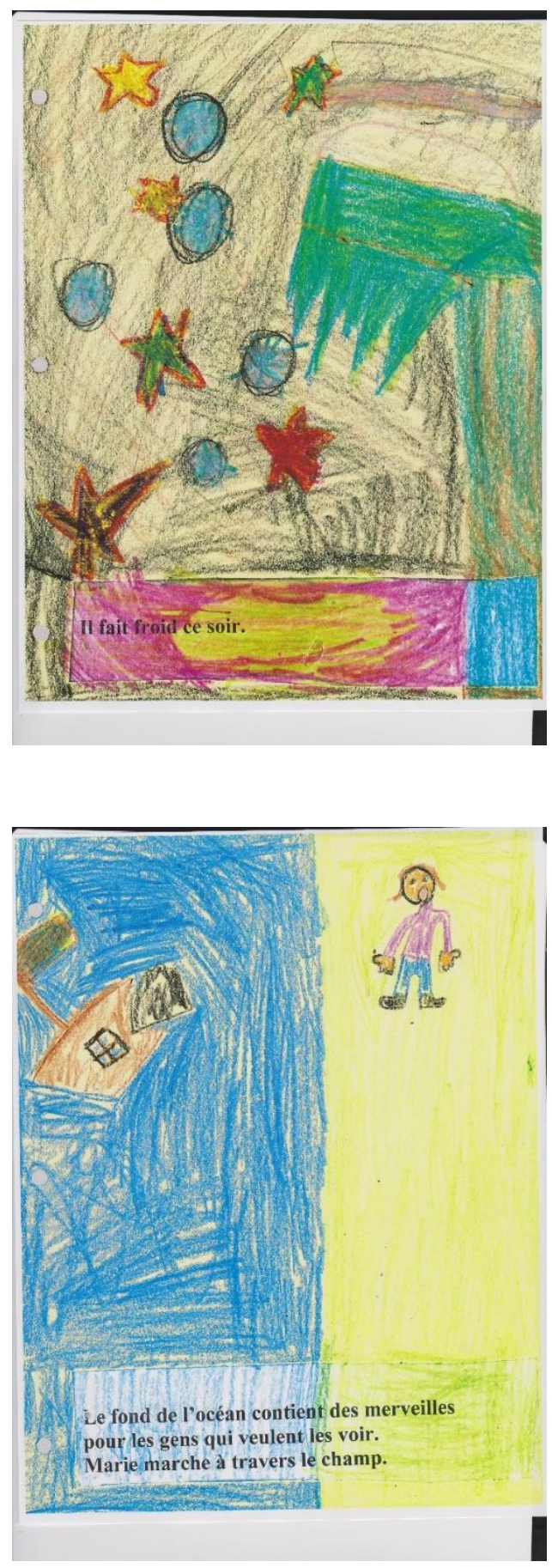



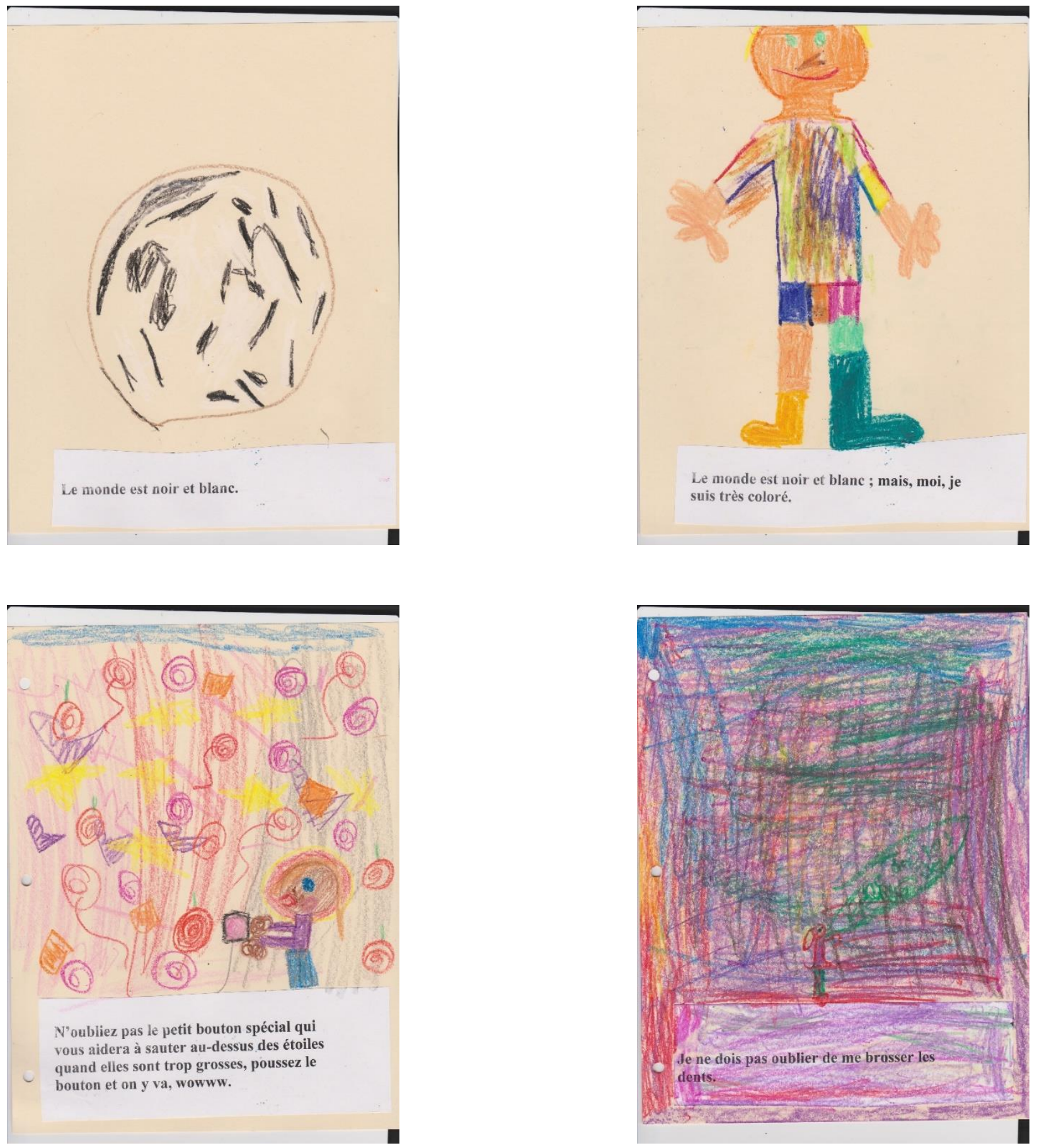


\section{Histoires pour enfants}

Auteurs : Leanna Ardron, Evan Arseneau, Taylor Ayres, Annette Beauchamp, Alyssa Berardocco, Meaghan Bisson, Stephanie Brule, Laura Burey, Tyler Cazes, Christina Ciantar, Sabrina Colella, Daniel Cursio, Julia Dimatteo, Kendall Farmer, Shawn Girard, Cassandra Gordon, Jaimee Gregoire, Kathryn Joron, Kelsey Kavanagh, Chelsea Kellner, Karly Knight, Madison Lucio, Maryann Mazuran, Adrianna Medyk, Alexis Merpaw, Sarah Mesquita, Alexandra Papadakis), Caterina Stambolic, Hannah Sukkau, Alyssa Tiangco, Melissa Timgren, Heather Turner, Alanna Vreman, Carole Webster, Rhiannon White

\section{(Transcription : Karly Knight)}

Les étoiles roses jouent dans les vagues qui viennent s'écraser.

Les étoiles blanches dansent sous la pluie rose.

Les étoiles font des sentiers à travers les feuilles bleues.

André le caméléon voit un singe. André le caméléon voit un chien, une femme qui crie,

les nuages, et un lit vert.

Tout le monde pense que le fond de l'océan est tout noir et triste.

Pierre LePoulpe pense différemment.

Le fond de l'océan contient des merveilles pour les gens qui veulent les voir.

Marie marche à travers le champ.

Elle cherche son éléphant qui se cache.

Il fait froid ce soir.

Les étoiles sont de sortie et on est entouré du noir de ciel.

Le lion rugit dans le ciel de la nuit.

Il rugit bonjour.

Lorsqu'Olivia a eu quatre ans, l'ours en peluche, Theo savait qu'ils seraient séparés pour la première fois. Olivia a dû aller à l'école comme tous les autres enfants de son âge et Theo n'était pas autorisé à l'accompagner.

Le jour approchait, Theo s'apprêtait a dire au revoir quand, tout a coup, Olivia le cacha dans son sac d'école.

Theo s'est retrouvé dans un bus,

Puis à l'école ...

Theo était heureux d'être avec Olivia. 
«Papa, pourquoi est-ce que les oiseaux volent?»

«Parce que les oiseux ont des ailes »

« Je veux voler»

«Oh, ma petite»

«Où est Bruno ? »

«Il dort devant le feu »

« Pourquoi est-ce qu'il dort»

« Parce qu'il est un chien et les chiens dorment beaucoup »

«Papa?»

«Oui, Sophie?»

«À l'école, j'ai appris que les ours dorment l'hiver »

« Oui, c'est vrai, et maintenant il est temps de ce coucher... Dis bonsoir à Maman »

«D'accord, Papa »

Dans l'obscurité de la nuit,

le vent d'hiver crie

les lampadaires vacillent,

les étoiles brillent.

Le clair de lune brille,

un visage apparaît.

Voici une femme habillée avec des fleurs,

du haut de la tête jusqu'à la pointe des orteils.

Gérôme la girafe adore les nœuds papillons.

Ce soir, c'est son $10^{\mathrm{e}}$ anniversaire.

Il essaie de trouver le nœud papillon parfait pour la soirée.

Mais, il ne peut pas choisir.

Il regarde les nœuds papillons bleu, rouge, jaune et vert.

Il ne peut pas choisir.

Gérôme a finalement décidé...

Qu'il va porter tous ses nœuds papillons.

Il était le mieux habillé de la soirée.

Le monde est noir et blanc.

Mais, moi, je suis très coloré. Mon cœur est rouge : plein d'amour pour ma famille, mes amis et toute nouvelle personne que je rencontre.

Mes yeux sont bleus comme l'océan profond.

Mes pieds sont verts parce que je passe mon temps à explorer.

Mon cerveau est jaune, plein d'idées brillantes. Le reste de mon corps est orange. Le monde est noir et blanc ; mais, moi, je suis très coloré. 
Lui, c'était un tout petit chaton.

Cerné de gros chats,

Il ne pouvait ni grimper, ni chasser les souris.

Quand il essayait de miauler,

Il poussait seulement un tout petit cri.

Tu ne grandiras jamais.

$\mathrm{Tu}$ ne pourras jamais grimper avec nous,

Ni chasser avec nous,

Tu ne seras jamais un chat,

Disaient-ils.

Mais un jour, le chaton a poussé un cri,

Qui n'était ni un miaulement,

Ni même un grognement,

Mais un rugissement,

Parce que le chaton était un lion.

Mademoiselle Rose est une professeure de dance qui enseigne la salsa aux enfants et aux adultes. Elle a un grand amour de la salsa et quand elle danse, ses cheveux deviennent bleus.

Plus Mademoiselle Rose danse, plus la couleur bleue remplit ses cheveux.

Ses cheveux dansent au rythme de la musique.

Petit Nicholas et sa maman vont au supermarché. En route, sa maman décide des arrêter à la station de lavage.

Soudainement, Petit Nicholas commence à pleurer. Il voit une voiture qui se fait avaler par un gros monstre!

Sa maman rassure Petit Nicholas ; tout ira bien. La voiture entre dans la station de lavage et Petit Nicholas couvre ses yeux.

Mais sa maman dit « Nicholas! Regarde ! Ouvre les yeux !» Il regarde et voit la voiture enveloppée de grosses bulles multicolores! Il sourit et essaye de faire éclater les bulles en mettent sa main sur la vitre. Nicholas crie encore, encore, encore !

Un jour, Rouge, Bleu et Jaune ont décidé de faire une peinture.

Mais Bleu et Jaune ne sont pas d'accord. Ils commencent à se battre.

Rouge ne sait pas que faire !

Soudainement, un petit morceau de Jaune et un petit morceau de Bleu se mélangent. Qu'est-ce que c'est? Vert a été créé !

Rapidement, Rouge frappe bleu et Violet d'inviter les couleurs secondaires !

Il était triste, le chien a la fourrière.

Au-dessus de sa tête, il y avait un nuage bleu de pluie.

Le jour que nous l'avons amené à la maison, je croyais qu'il ne sourirait jamais. Mais au moment où je lui ai montré son nouveau lit et de nombreux jouets, le nuage a disparu. 
Il a remué sa queue et m'a donné des baisers.

Depuis ce jour-là, nous sommes les meilleurs amis.

Ce matin, mon réveil n'a pas sonné.

Je vérifie le temps et je suis presque en retard pour l'école.

Je ne dois pas oublier de me brosser les dents.

Je ne dois pas oublier de dire au revoir à maman.

Je cherche mes parents. Ils dorment encore !

Je cours vers l'arrêt de bus.

Je vois le bus. Il reste stationné.

Je m'approche... le moteur rugit... les roues commencent à rouler... et le bus s'en va.

Comme je commence à pleurer, je vois le chauffeur. Il ouvre la fenêtre. Que dit-il ?

«Mon ami, nous sommes samedi!»

AHHHH ! Je me réveille de mon sommeil. J'ai chaud et je transpire et mon cœur bat rapidement.

Je veux courir au lit de mes parents, mais je ne peux pas.

Je ne peux pas parce que mon lit est au milieu de l'océan.

C'est le milieu de la nuit, c'est sombre et mon lit flotte dans l'océan.

Et ensuite, du coin de mon oeil, je le vois.

Un requin !!

Je ne peux pas quitter ce lit. Le requin nage autour rapidement.

Il fait des bruits effrayants et montre ses dents pointues. Je dois m'endormis ; ainsi je peux me réveiller pendant un meilleur rêve.

Venez, venez vit, on va où personne n'est jamais allé. Apportez vos bicyclettes, vos espadrilles et mettez vos casques.

Faites-attention ! Il faut toujours regarder à droite et à gauche avant de traverser les étoiles. N'oubliez pas le petit bouton spécial qui vois aidera à sauter au-dessus des étoiles quand elles sont trop grosses, poussez le bouton et on y va, wowww.

Oh non, nous avons perdu le professeur. Il fait le trouver pour retourner à la maison, sinon, nous devrons rester ici.

Les montagnes voient tout.

Fait dodo mon petit, il est temps d'aller te coucher. Ne te fâche pas, voici ton ourson favori. Ne pleure pas, je te bercerai très doucement.

$\mathrm{Ne}$ crie pas, je te chanterai une petite chanson mélodieuse. Fais do-do mon petit, c'est le temps d'aller te coucher.

L'école d'Émile accueille un défilé de monde. Les élèves ont reçu l'invitation. Ravi, Émile est un des premiers à s'inscrire.

En marchant dans la ville, il voit dans de petites boutiques des matériaux qu'il aimerait utiliser pour ses vêtements. 
Il fabrique une robe un papier brun avec des manches à volants, trois colliers et une broche gigantesque composée de pétales de fleurs.

Quand Émile a avancé sur le podium, les spectateurs applaudissent et Émile a gagné !

Gustave vit à Paris en France.

Il veut être à la mode.

Gustave passe ses journées à flâner dans les rues pour que tous voient ses habilles.

Malheureusement, il n'a aucun ami avec qui parler. Il est trop occupé par sa propre personne.

«Peut-être, pense Gustave, qu'être à la mode n'est pas si important que ça. »

Bonjour, je m'appelle Lila, j'ai quatre ans.

Voici mon ourson favori, il s'appelle Tutu.

Il est violet avec des taches vertes, bleues, et blanches.

Je prends Tutu partout. Il vient avec moi à l'école, en vacances, à la maison de mes grandsparents et il est là quand je dors dans mon lit.

Je t'aime, Tutu.

Un lundi matin chaud et ensoleillé, Mélanie se leva d'un long sommeil. C'était le premier jour des vacances d'été et elle était très heureuse de ne pas aller à l'école.

Son père a préparé son petit déjeuner préféré : des crêpes avec beaucoup, beaucoup, beaucoup de pépites de chocolat.

«Merci papa! Maintenant, je peux aller jouer dehors avec mes amis ? »

Papa a déclaré : «Ma fille, qui va m’aider à nettoyer ?»

J'aime nager dans l'océan que mes meilleurs amis y habitent. Chaque jour je vais à la plage et je trouve sous l'eau le corail coloré.

C'est l'endroit où les tritons et les sirènes aiment jouer. Nous explorons la caverne bleue l'aprèsmidi et nous dansons avec les poissons heureux.

Maman ne croit pas à mes histoires de la mer... Elle dit toujours « Quelle imagination!» 